\title{
BMJ Open Inhaler technique education in elderly patients with asthma or COPD: impact on disease exacerbations - a protocol for a single-blinded randomised controlled trial
}

Tiago Maricoto, ${ }^{1,2}$ Jaime Correia-de-Sousa, ${ }^{3,4}$ Luís Taborda-Barata ${ }^{5,6}$

To cite: Maricoto T, Correiade-Sousa J, Taborda-Barata L. Inhaler technique education in elderly patients with asthma or COPD: impact on disease exacerbations-a protocol for a single-blinded randomised controlled trial. BMJ Open 2019;9:e022685. doi:10.1136/ bmjopen-2018-022685

- Prepublication history and additional material for this paper are available online. To view these files, please visit the journal online (http://dx.doi. org/10.1136/bmjopen-2018022685).

Received 1 March 2018 Revised 10 November 2018 Accepted 20 November 2018

Check for updates

(C) Author(s) (or their employer(s)) 2019. Re-use permitted under CC BY-NC. No commercial re-use. See rights and permissions. Published by BMJ.

For numbered affiliations see end of article.

Correspondence to

Dr Tiago Maricoto;

tiago.maricoto@gmail.com

\section{ABSTRACT}

Introduction Chronic Obstructive Pulmonary Disease

(COPD)and asthma affect more than $10 \%$ of the population. Most patients use their inhaler incorrectly, mainly the elderly, thereby becoming more susceptible to poor clinical control and exacerbations. Placebo device training is regarded as one of the best teaching methods, but there is scarce evidence to support it as the most effective one to improve major clinical outcomes. Our objective is to perform a single-blinded RCT to assess the impact of this education tool in these patients.

Methods and analysis A multicentre single-blinded Randomised Controlled Trial (RCT) will be set up, comparing an inhaler education programme with a teachto-goal placebo-device training versus usual care, with a 1-year follow-up, in patients above 65 years of age with asthma or COPD. Intervention will be provided at baseline, and after 3 and 6 months, with interim analysis at an intermediate time point. Exacerbation rates were set as primary outcomes, and quality of life, adherence rates, clinical control and respiratory function were chosen as secondary outcomes. A sample size of 146 participants (73 in each arm) was estimated as adequate to detect a $50 \%$ reduction in event rates. Two-sample proportions $\chi^{2}$ test will be used to study primary outcome and subgroup analysis will be carried out according to major baseline characteristics.

Ethics and dissemination Every participant will sign a written consent form. A Data Safety Monitoring Board will be set up to evaluate data throughout the study and to monitor early stopping criteria. Identity of all participants will be protected. This protocol was approved on 22 November 2017 by the local Ethics Committee of University of Beira Interior, with the reference number CEUBI-Pj-2017-025. Results will be presented in scientific meetings and published in peer-reviewed journals. Trial registration number NCT03449316; Pre-Results.

\section{INTRODUCTION}

Epidemiology

Asthma and COPD affect about $10 \%$ of the population, but many patients have uncontrolled symptoms. ${ }^{1}$ In asthma, in particular,
Strengths and limitations of this study

- This study is innovative because it includes exclusively elderly patients with asthma or COPD, addressing, in a 1-year follow-up, a specific placebo device education programme, alone, without any other aspects

- No previous study has addressed this teaching method in these patients, as it seems to be the most efficient one.

- Our study has a randomised design, which has been a major limitation in previous studies.

- The 1-year follow-up period, with one interim evaluation, allows this study to comprehensively address the real impact of a regular education programme.

- The main limitation of this study is the single blinded design, due to the nature of intervention itself, which may introduce some performance bias.

it should be highlighted that only $57 \%$ of all patients were shown to have their symptoms controlled, ${ }^{23}$ and the elderly population is particularly vulnerable to this condition. ${ }^{3}$ In fact, late onset asthma may be frequently misdiagnosed and mistreated, and the risk of drug interactions also requires close monitoring. ${ }^{4}$ Hospitalisation rates due to asthma and COPD are reported to reach $27 \%$ among non-adherent patients and could be up to $53 \%$ in community treated cases, and this may be even more apparent in elderly patients. It should also be stressed that good adherence to inhaler treatment may, in contrast, be associated with a lower rate of severe exacerbations, with reductions observed in up to half of the cases. ${ }^{5-7}$

\section{Inhaler technique}

Inhaled therapy is the most widely used way to treat patients with asthma and COPD, ${ }^{8}$ but up to $90 \%$ of them do not use their inhalers correctly. ${ }^{9}{ }^{10}$ Performance errors have been 
described with almost every type of device, and over the past decades this problem has not improved, which highlights the need to better understand the specificities of different inhaler use as well as the impact of different inhaler teaching methods. ${ }^{11}$ Several inhaler devices are available on the market and it seems that differences either in device type or in patient characteristics may significantly influence performance. ${ }^{12}$ However, all inhalers, when properly used, show no significant differences in terms of treatment efficacy, ${ }^{13}$ but it is well established that poor inhaler technique leads to poor clinical control ${ }^{1516}$ and also to increased health costs. ${ }^{17}$ In addition, some type of specific errors seem to have a higher impact on clinical control, but there is no consensus yet on which errors are critical and non-critical. ${ }^{1819}$

Patients in controlled trials receive more training in inhaler performance and more counselling on adherence than patients who are seen as part of routine clinical practice, but few studies have addressed these variables as separate outcomes. ${ }^{20}$ Some studies show that teaching inhaler technique may lower the risk of exacerbations and death. ${ }^{61}{ }^{22}$ However, its impact is quickly lost as time elapses, suggesting this is a practice that should be rechecked and regularly applied to patients. ${ }^{23}{ }^{24}$ Nevertheless, how often the review should be carried out has not been established yet, since most studies have not addressed this issue in an isolated manner.

Significant evidence has shown that inhaler technique performance is regarded as particularly complex by older patients. ${ }^{25} 26$ These patients also present lower adherence rates $^{9}$ and are more resistant to correct performance. ${ }^{27} 28$ Furthermore, other major characteristics may influence inhaler use, such as educational level, previous teaching or even age itself (ie, age above 75 years).$^{29}$ However, the significance of these observations still has to be fully ascertained since elderly patients are frequently excluded from major clinical trials. Randomised studies with elderly patients are scarce, and most of them did not address these aspects. Some of these studies have shown significant reductions in exacerbation risk, but most of them addressed several aspects of intervention besides inhaler technique education itself, namely self-management plans, disease knowledge, management of exacerbations and their triggers. None has yet addressed inhaler review alone or in a regular education programme..$^{2130-33}$

Inhaler technique may be taught using many tools, such as step-by-step flyer schemes, video demonstrations, videoconferencing and face-to-face demonstrations or even using web-based platforms, but there is insufficient evidence about which is the best education method to improve inhaler performance or its impact on major outcomes. ${ }^{34-37}$ Nevertheless, some studies including adult patients as well suggest that the most efficient method seems to be using a teach-to-goal approach with placebo device demonstration and training provided in person. ${ }^{38-42}$ In addition, manufacturers' recommendations often differ from clinical guidelines, which makes it difficult for patients to fully understand all the necessary steps of inhaler use. ${ }^{43}$ This highlights the importance of watching patients using their inhalers, which can be achieved with a placebo device training set.

This study will focus on elderly patients and aims at testing the effect of a structured and regular placebo device training approach on disease exacerbation rates.

\section{SPECIFIC AIMS AND HYPOTHESES}

Our objective is to test the impact of an inhaler technique education programme on the risk of exacerbations in elderly patients with asthma or COPD.

The main hypothesis is that, among elderly patients with asthma or COPD, regular education of inhaler technique using a teach-to-goal placebo device-based approach, and delivered by family doctors at baseline, 3 and 6 months, can reduce the exacerbation risk by $50 \%$ after a 1 year follow-up, when compared with usual care.

\section{RESEARCH DESIGN AND METHODOLOGY Study design}

Two arms single blinded randomised controlled trial with a 1-year follow-up (figure 1). Participants will be allocated to each group on a random basis, which is defined by a computerised generator and is independent of the control of the principal investigator. The allocation sequence of the 146 participants will be defined through a computer generator prior to the start of the study. After the generation of this sequence, 146 envelopes will be created, numbered in the appropriate order and will contain the result of the allocation. The order of the envelopes' number will define the order of participants' enrolment. The principal investigator will not be aware of the information contained within the envelopes, thereby maintaining a minimisation randomisation process. To ensure the accuracy of the use of the envelopes, the documents inside the envelope will be signed by the Data Safety Monitoring Board and must be returned by the researchers after the allocation of the participants.

\section{Sample size calculation}

Sample size was estimated using the $\chi^{2}$ independent group proportions approach of STATA Statistical Package, considering the event proportion in control group of $50 \%(0.5$ annual rate) as reported in other previous studies ${ }^{21} 2244$ and estimating a reduction of event rate in the intervention group to $25 \%$ ( 0.25 annual rate) as reported in similar studies. A 95\% CI, with value (power) of $80 \%$, an alpha level of $5 \%$ and a ratio of cases/controls of 1:1 were established. Finally, the sample size was readjusted upward, considering an estimated proportion of full compliance of the study of $80 \%$ (20\% losses). The estimated sample size was 116, readjusted to a total of 146 individuals (73 in each arm). 


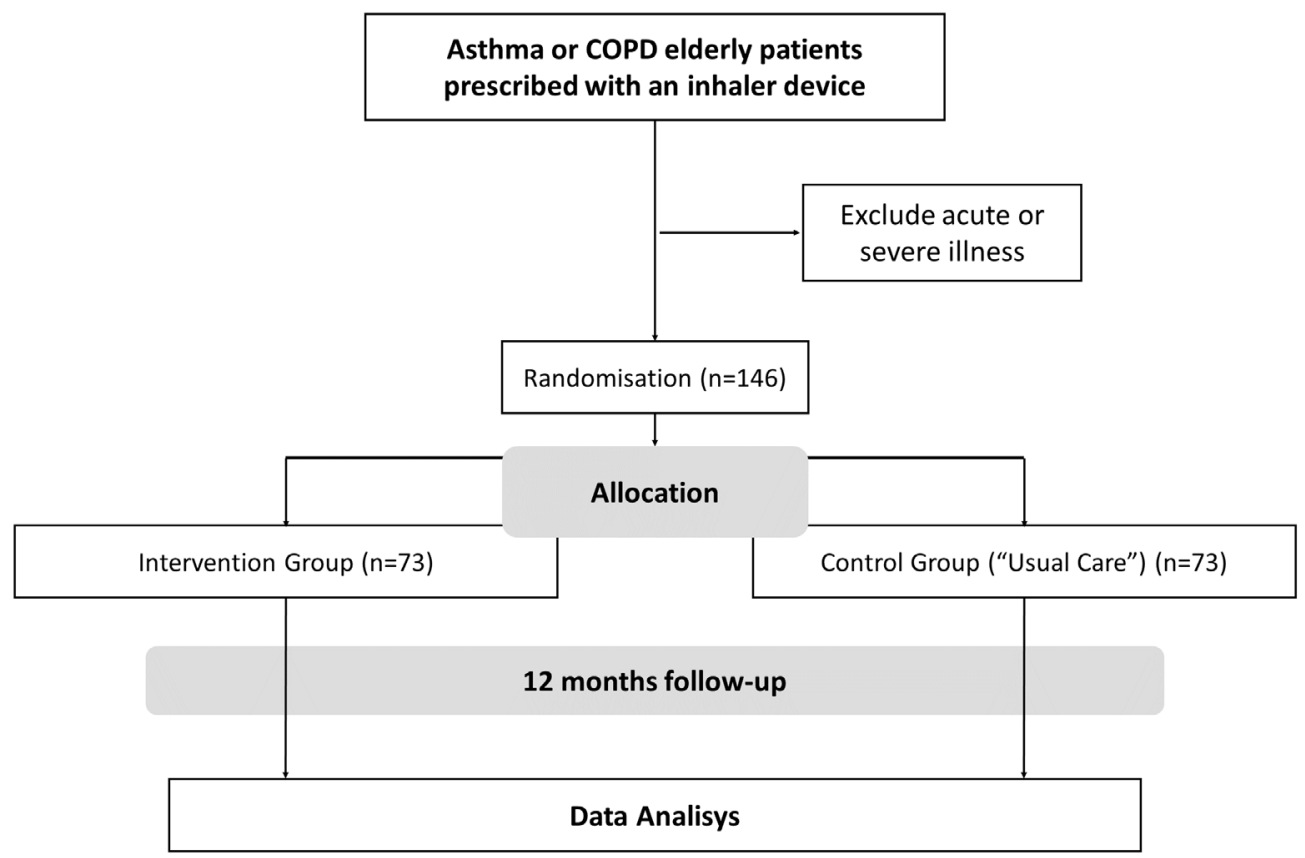

Figure 1 Study design diagram.

\section{Inclusion criteria}

Patients with a diagnosis of COPD or asthma, prescribed any kind of inhaler device (pressurised Metered Dose Inhaler (pMDI) with or without Spacer, Dry Powder Inhaler or Soft Mist), aged $\geq 65$ years and being a regular user of primary healthcare services (defined as having had at least one appointment in the last 2years with his/her own Family Doctor). In order to minimise diagnostic inaccuracy, asthma and COPD diagnosis will be reviewed in every participant at baseline prior to enrolment and in accordance with GINA and GOLD strategies. ${ }^{456}$

\section{Exclusion criteria}

Severe or acute illness (such as unstable cardiovascular status, unstable angina, recent myocardial infarction (within 1 month) or pulmonary embolism, haemoptysis of unknown origin, recent pneumothorax (within 1 month), recent thoracic, abdominal or eye surgery (within 1 month), acute nausea or vomiting, severe respiratory distress, dementia).

We will exclude patients who do not need inhaler medication on a daily basis, since these patients are less susceptible to the full impact of the intervention. In addition, these are mostly patients with intermittent asthma as well as patients with COPD with mild obstruction (GOLD stage I) and tend to have a low frequency of disease exacerbations, which would hamper our ability to detect a true outcome effect.

\section{Predictors/Intervention}

Intervention Group: This group will receive a structured and regular follow-up plan, with education on inhaler technique. Patients will be trained by a Family Doctor (the primary investigator) in terms of the inhaler technique using placebo devices similar to their own devices. We will start by evaluating their baseline technique, and then, a teach-to-goal approach will be used with correction of identified errors. Then, we will ask patients to demonstrate the inhaler technique, and again, committed errors will be corrected by demonstration. We will repeat all correct steps as many times as needed in order for patients to perform them correctly. This intervention will be performed at baseline, 3 and 6 months. Outcomes will be assessed at baseline and after 3, 6 and 12 months, since there is dissenting evidence about the best timeline to achieve significant exacerbation risk reductions..$^{21} 3032$ In each visit, and prior to the main intervention with the primary investigator, assessment of the inhaler technique and application of all questionnaires (clinical control, treatment adherence and quality of life) will be performed by a secondary blinded investigator.

Control Group: This group will receive usual care from their own Family doctors, with no specific intervention. Each doctor will perform the necessary clinical appointments according to his/her real life judgement. Besides this, this group will have visits at baseline and after 3, 6 and 12 months to assess secondary outcomes. At each visit, assessment of the inhaler technique and application of all questionnaires (clinical control, treatment adherence and quality of life) will be performed by a secondary blinded investigator. At any appointment, if the patient asks for or if the clinician decides to teach inhaler technique, that will be recorded, since it will be important to analyse and control for the true effect size of intervention.

If any adjustments are made in drug classes or device types in any participant, this information will be recorded.

\section{Outcomes of interest}

Primary outcome

Adverse events (continuous, time to event). 
For asthma, an event will be defined as increased respiratory clinical symptoms leading the patient to search for medical care and resulting in any of the following:

- Need for increased inhaled corticosteroid dose of at least $4 \times$ the regular dose.

- Need for increase of short-acting ${ }_{2}$ agonists on a daily basis.

- Need for oral corticosteroids.

- Need for oral antibiotics.

- Hospitalisation or emergency room (ER) visit with increased respiratory clinical symptoms.

For COPD, an event will be defined as increased respiratory clinical symptoms prompting the patient to search for medical care, and resulting in any of the following:

- Need for increase of long-acting ${ }_{2}$ agonists on a daily basis.

- Need for oral corticosteroids.

- Need for oral antibiotics.

- Hospitalisation or ER visit with increased respiratory clinical symptoms.

Respiratory-related mortality and all-cause mortality will also be considered an adverse event.

All adverse events and mortality causes will be carefully analysed in order to assess their eligibility by two independent and external investigators, who will constitute a Data Safety Monitoring Board. This will be performed using different platforms of clinical records, from the ER of the regional reference hospital, from the Primary Healthcare facilities (such as PEM for prescribed drugs, SCLINICO for clinical records and PDS for ER records) and even by asking the participant for additional information. After any event, and if necessary for ethical reasons, inhaler technique and adherence improvement will be addressed by the primary investigator regardless of the participant allocation, and in accordance with the recommendation of the Data Safety Monitoring Board.

\section{Secondary outcomes}

- Clinical assessment using COPD Assessment Tools and modified Medical Research Council for COPD; Control of Allergic Rhinitis and Asthma Test ${ }^{47}$ and Asthma Control Test for asthma. ${ }^{48}$

- Quality of Life using St. George's Respiratory Questionnaire $^{49}$ and Clinical COPD Questionnaire ${ }^{50}$ for COPD and Asthma Quality of Life Questionnaire. ${ }^{51}$

- Functional control using Forced Expiratory Volume in $1 \mathrm{~s}$ (FEV1), forced vital capacity (FVC), peak expiratory flow and maximum expiratory flows of $25 \%-75 \%$ of FVC (MEF25-75) as a \% of predicted value; and FEV1/FVC ratio.

- Adherence rate using the Brief Medication Questionnaire (this will also evaluate the frequency of using the devices).$^{52}$

- Number of errors in inhaler technique (that will be standardised to a score up to $100 \%$ scale).

(To evaluate inhaler technique performance with each device, the Aerosol Drug Management Improvement Team (ADMIT) protocols and guidelines will be used, ${ }^{53}$ evaluating all the recommended steps for inhaler use in each one of them (pMDI with or without chamber, Qvar Autohaler, Turbohaler, Diskus, Aerolizer, Handihaler, Breezhaler, Novolizer, Genuair, Twisthaler and Easyhaler). For those devices that do not have any protocol from the ADMIT group we will use the recommendations from the manufacture`s Summary of Product Characteristics (Soft Mist Inhaler, Budesonide from Farmoz, Ellipta, Spiromax and Forspiro)) .

All questionnaires will be used in validated Portuguese versions. ${ }^{47-525455}$ All participants will perform spirometry with bronchodilation test at baseline visit for diagnostic confirmation, as well as a baseline spirometry without bronchodilation for functional control at subsequent visits. A certified provider will perform spirometry.

\section{Other variables collected at baseline}

- Demographics (body mass index, age, sex).

- Classification of clinical status, according to:

- Exacerbation history.

- Years of diagnosis.

- Asthma classification/stage according to GINA guidelines (clinically as well controlled, partially controlled or uncontrolled; and therapeutically as in STEP $1,2,3,4$ or 5$).^{45}$

- COPD stage according to 2017 GOLD guidelines (combined assessment stages A, B, C and D; and severity of airflow limitation GOLD 1, 2, 3 and 4$).{ }^{46}$

- Social class according to Graffar classification (Portuguese version).$^{56}$

- Comorbidities (such as concomitant allergic rhinitis, cancer, cardiac heart failure, alcohol or drug abuse, current smoking and smoking pack years, diabetes mellitus, previous stroke or acute myocardial infarction, thoracic, abdominal or cerebral aneurysms, severe osteoarthrosis in hands and upper limbs).

- Depression using Geriatric Depression Scale in Portuguese. ${ }^{57}$

- Frailty state in elderly, using a self-reported instrument in Portuguese. ${ }^{58}$

- Cognitive function using Montreal Cognitive Assessment (MOCA) in Portuguese. ${ }^{59}$

- Influenza and pneumococcal vaccination status.

- Previous teaching of inhaler technique, specifying the education type (placebo device, video, leaflet, multimedia and so on).

- Years of use with current device.

The principal investigator will collect all baseline data prior to allocation and randomisation, and this will be recorded in a proper form.

\section{Statistical analysis}

The hypothesis testing approach will be the following:

Null hypothesis

Teaching inhalation technique performance with a placebo device approach does not reduce the exacerbation 
risk in elderly patients with asthma or COPD after a 1-year follow-up.

\section{Alternative hypothesis}

Teaching inhaler technique performance with a placebo device approach reduces the exacerbation risk in elderly patients with asthma or COPD after a 1-year follow-up.

\section{Dichotomous predictor}

Usual Care VS Regular teach-to-goal education with placebo device.

\section{Dichotomous outcome}

Exacerbation: Yes/No.

Data will be analysed using the STATA Statistical Package ${ }^{\odot}$ software.

\section{Test statistic for primary outcome}

Dichotomous data will be analysed with a two-sample proportions $\chi^{2}$ test and a COX proportional hazard timeto-event analysis, and both arms will be compared using the measures of association: risk ratio; risk difference; HR and number needed to treat analyses.

\section{Test statistic for secondary outcomes}

Continuous data will be analysed using parametric tests, such as $\mathrm{T}$ test for comparison of mean values and dichotomous data will be analysed using $\chi^{2}$ test. In order to test differences between groups in the mean values of continuous analysis, mixed effects models for repeated measures will be used. For binary outcomes, linear regression models with group-time interactions will also be adapted, and generalised linear models (such as Poisson regression) will be applied for exacerbations, as recommended in the literature. ${ }^{60}$ As an alternative approach, generalised estimating equation models will be used to handle unmeasured dependence between outcomes.

In case of cohort losses above $20 \%$, comparative analysis for intention to treat, per-protocol and a multidata imputation will be carried out. Missing data will be treated as missing completely at random. Subgroup analysis will be performed according to secondary variables, such as diagnosis, age (including stratification into the following categories: $65-75,75-85$ and $>85$ years), sex, years of diagnosis, disease classification/stage, comorbidities, educational level, previous teaching of inhaler technique, device type as well as the specific types of detected errors (in order to identify the most critical ones). This will be performed using regression models to multivariate analyses.

An interim analysis will be performed midway through the follow-up, namely at 6 months, defining a significance level adjusted by the Bonferroni technique of $0.025 .{ }^{61}$

\section{Study setting}

The study will be conducted in a multicentre network that will include two or three primary care centres, which will be coordinated by a team of experts in the field. All of them will be in urban or suburban areas. A Portuguese primary care centre usually accounts approximately for more than 10000 patients, and about $30 \%$ of them are aged above 65 years. Considering an approximate prevalence of asthma and COPD of $8 \%$ in this population, there is a potential target population of almost 250 patients in each healthcare facility. Recruiting patients at more than one site will improve the feasibility, reproducibility and credibility of the study, but will increase all the logistic issues.

All invited participants will have a first contact will the primary investigator to confirm the diagnosis and all the eligibility criteria, and to carefully explain all the study procedures before their inclusion and subsequent randomisation. Diagnosis will be confirmed according to state of the art and the previously mentioned updated guidelines and with spirometry. The number of patients screened and deemed ineligible as well as the number of patients who are considered eligible but decline participation will be also recorded.

\section{Timeline}

- Study protocol final version: August 2017.

- Ethics consent and scientific academic authorisation: December 2017.

- Clinical administrative authorisations: first semester of 2018.

- Multicentre team gathering: first semester of 2018.

- Beginning of recruitment: second semester of 2018.

- End of recruitment: second semester of 2019.

- Data analysis and dissemination: during 2020.

\section{Patient and public involvement}

No patient or public were involved in the design of this protocol, or in the establishment of the intervention and the outcome measures. Results from all participants will be given to their own family doctors in order to be used if deemed necessary to clinical practice.

\section{DISCUSSION}

This study is innovative because it includes exclusively elderly patients with asthma or COPD, addressing a specific placebo device education programme, alone, without any other aspects, and it was designed to detect a significant reduction on disease exacerbation rate. It is expected to detect approximately 55 adverse events, 18 in the intervention group and 37 in the control group. In addition, it is expected to find a more significant improvement in the intervention group, in all clinical and functional parameters during the follow-up.

This study has some limitations, mainly in selection bias due to the risk of missing data and follow-up losses. To overcome this problem, different strategies will be applied, such as an increase in estimated sample size, readjusted for an estimation of $20 \%$ losses and sending a reminder prior to each visit using SMS/Email/Call to contact the participant. 
Another aspect that could bias our study is the Hawthorne effect throughout the study (ie, behaviour change in participants due to their involvement in the study). However, we believe that by establishing a cohort time of 1 year, this effect will not be sustained. On the other hand, the control group ('usual care') will maintain their usual care at their own family doctors, who are completely free from any influence of the study design. For this reason, the control group ('usual care') participants will not receive any intervention from the primary investigator. They will only contact with the secondary investigator in order to collect endpoints and outcome data, and the latter is completely blinded to randomisation. With this approach, the Hawthorne effect will not contaminate the control group and will represent a real life usual care. On the other hand, the Data Safety Monitoring Board will be composed of two external investigators, who will, together with the statistician, be blinded to the endpoints and outcomes (PROBE setting). Using usual care as the comparator arm also brings some limitations to consider, because it is not a perfect comparator due to its nature. It is not sufficient for good patient outcomes and it is not standardised. This aspect is due, for instance, to the fact that patients on usual care can receive interventions on inhaler education and self-management tools from other uncontrolled sources. To overcome that we will retrospectively query patients in this arm and their own family doctor for any type of interventions that may have been delivered during the study period.

Another possible limitation of our study is that we will not use electronic measures of adherence and inhalation techniques. These are a very useful approach to monitoring real world adherence to inhaler therapy. In fact, these electronic measures overcome the bias seen with self-report and other problems observed with objective medication checks such as prescription refill rates. However, most electronic measures of adherence do not measure timing of device activation but rather the overall number of activations performed, and, in addition, this measure does not mean that medication was taken on a regular basis (patients may just activate the inhaler several times, prior to handing over the device). It is not until recently that a new device has been studied, which seems to overcome this problem, and which also analyses inhaler technique, but it is not widely available-INCA device. ${ }^{62}$ Nevertheless, these devices are expensive and their use could not be implemented in our study. We therefore decided to use the adherence questionnaire (BMQ), which is a well-validated tool in several languages worldwide and also in Portuguese.$^{52}$ Furthermore, it is a very simple and easy method to detect non-adherence, which also allows separating subdomains of adherence. Thus, it is a good tool for assessing adherence in our study involving the general population of patients with asthma and COPD. Regarding inhalation technique, we decided to use regular checklists, since they are the most widely method used in other studies, thereby allowing further comparisons. They are also easy to use and allow detection of critical errors in each device.

The standardisation of the protocol intervention is another issue to be considered. In order to overcome different approaches among different investigators from different multicentre sites, a protocol with detailed instructions will be created to guide them during the intervention (investigators) and assessment visits (secondary investigators). This protocol will explain all the steps and procedures for training inhaler technique as well as for assessing it, and all the procedures to follow in each visit for assessing the outcomes.

Primary investigators will be trained in communication techniques related to inhaler education of different devices and all of them will have a kit of placebo devices for use with participants. Such training will allow the standardisation of all procedures of intervention and it will be provided ahead by the coordination team of the study.

\section{Ethics and dissemination}

The study protocol has already been analysed by the local Ethics Committee of University of Beira Interior, with the reference number CE-UBI-Pj-2017-025 and was approved on 22 November 2017.

Every participant will sign a written consent form (online supplementary appendix I). We decided to use 'usual care' as the main comparator instead of another intervention method, since all interventional methods have shown some degree of efficacy in clinically relevant outcomes, as previously mentioned. We thus believe that comparing with other education methods would minimise the effect detection of our teach-to-goal placebo-device intervention. Moreover, all of the randomised studies that included mostly elderly patients also used 'usual care' as a comparator, which will be important when comparing them with our results. However, we highlight the fact that those studies did not use the same age criteria as we are using, since they also included non-elderly adult patients in their samples. In addition, they did not just focus on inhaler teaching, since they provided additional sessions with other programme elements, such as self-management care. There is, thus, insufficient evidence about the efficacy of inhaler education as an isolated intervention, and for that reason, our approach will be novel and will significantly contribute towards clarifying those issues.

A Data Safety Monitoring Board will be set up, composed of two external investigators with a board expertise in this clinical field and in academic and scientific activities, to evaluate data obtained throughout the study. Evaluations will occur every 6 months, whatever the number of participants enrolled or the follow-up time reached at that point. The stop earlier criteria will be defined as any moment on follow-up in which the collected data show statistically significant differences in the primary outcomes. The study may be suspended earlier if sufficient data are obtained for at least 6 months of follow-up or if significant evidence of intervention effectiveness is 
obtained, providing that statistical significance values are met by the Bonferroni adaptation.

Invited participants who refuse to participate will be evaluated at baseline, according to previously mentioned characteristics, in order to compare them with the included cohort. They will also be invited to sign a written informed consent form that will allow investigators to collect such data. The documents used to collect the data of the participants will contain only an identification code of each participant, in order to protect their identity. The code of each participant must be composed of the initials of the first two names, followed by the last two digits of the National Healthcare Service Number (eg, Name FirstSurname SecondSurname, $123456789 \longrightarrow$ code 'NF89').

The number of participants considered ineligible will be recorded, as well as the number of eligible participants who refuse to participate in the study.

The results obtained from this study will be published in peer-reviewed journals and presented at scientific meetings of primary healthcare and respiratory fields. All data recorded during the study will be stored for a period of 5 years, in accordance with the Portuguese Clinical Research Law, in a safe and proper place in the primary investigator`s health centre. After this period, all data that contain participants' codes will be destroyed.

\section{Author affiliations}

${ }^{1}$ Aveiro-Aradas Family Health Unit, Aveiro Health Centre, Aveiro, Portugal

${ }^{2}$ Faculty of Health Sciences, University of Beira Interior, Covilhã, Portugal

${ }^{3}$ Life and Health Sciences Research Institute (ICVS)/3B's—PT Government Associate

Laboratory, University of Minho, Braga, Portugal

${ }^{4}$ Horizonte Family Health Unit, Matosinhos Health Centre, Matosinhos, Portugal ${ }^{5}$ CICS - Health Sciences Research Centre; NuESA-Environment \& Health Study

Group, Faculty of Health Sciences, University of Beira Interior, Covilha, Portugal

${ }^{6}$ Department of Allergy \& Clinical Immunology, Cova da Beira University Hospital Centre, Covilhã, Portugal

Acknowledgements The authors endorse acknowledgment to Professor Jonh Groarke from the Harvard Medical School for his important scientific support and input in reviewing the final version of this manuscript.

Contributors All authors have equally contributed to the elaboration of this protocol in every stage of its design and writing. TM elaborated the first draft of the study, JC-d-S and LT-B gave inputs to all necessary design adjustments, and all authors have carried out final revisions of the manuscript.

Funding This work was developed without any funding support or financial source. The academic affiliation of this protocol is the the Faculty of Health Sciences at the University of Beira Interior and Life and Health Sciences Research Institute (ICVS)/3B's at University of Minho in Portugal. This work was prepared with scientific support from Harvard Medical School, in accordance with the Portuguese Clinical Scholarship Research Training Program.

Competing interests JC-d-S reports competing interests from Harvard Medical School, during the conduct of the study, as scientific support. He also reports from Boheringer Ingelheim and from AstraZeneca outside the submitted work, and all these fees were received by a non-profit organisation to be used in CME and research. The remaining authors declare only from Harvard Medical School, during the conduct of the study, as scientific support.

Patient consent Not required.

Ethics approval Ethics Committee of University of Beira Interior, Covilhã, Portugal.

Provenance and peer review Not commissioned; externally peer reviewed.

Data sharing statement All data from the trial will be kept in a safe place of the principal investigator's institutional facilities and by the Data Safety Monitoring Board, in accordance with the national and international clinical research policies.
Open access This is an open access article distributed in accordance with the Creative Commons Attribution Non Commercial (CC BY-NC 4.0) license, which permits others to distribute, remix, adapt, build upon this work non-commercially, and license their derivative works on different terms, provided the original work is properly cited, appropriate credit is given, any changes made indicated, and the use is non-commercial. See: http://creativecommons.org/licenses/by-nc/4.0/.

\section{REFERENCES}

1. World Health Organization. The global burden of disease: 2004 update. 2008:160 http://www.who.int.

2. Sa-Sousa A, Morais-Almeida M, Azevedo LF, et al. Prevalence of asthma in Portugal - The Portuguese National Asthma Survey. Clin Transl Allergy 2012;2:15

3. de Sousa JC, Santo ME, Colaço T, et al. Asthma in an urban population in Portugal: a prevalence study. BMC Public Health 2011;11:347-47.

4. Reddel H, Baterman E, Becker A, et al. Global Strategy for Asthma Management and Prevention: Global initiative for asthma, 2015:149. http://www.ginasthma.org

5. van Boven JF, Tommelein E, Boussery K, et al. Improving inhaler adherence in patients with chronic obstructive pulmonary disease: a cost-effectiveness analysis. Respir Res 2014;15:66.

6. Vestbo J, Anderson JA, Calverley PM, et al. Adherence to inhaled therapy, mortality and hospital admission in COPD. Thorax 2009;64:939-43.

7. Engelkes M, Janssens HM, de Jongste JC, et al. Medication adherence and the risk of severe asthma exacerbations: a systematic review. Eur Respir J 2015;45:396-407.

8. Rees J. Methods of delivering drugs. BMJ 2005;331:504-6.

9. Cochrane MG, Bala MV, Downs KE, et al. Inhaled corticosteroids for asthma therapy: patient compliance, devices, and inhalation technique. Chest 2000;117:542-50.

10. Lavorini F, Magnan A, Dubus JC, et al. Effect of incorrect use of dry powder inhalers on management of patients with asthma and COPD. Respir Med 2008;102:593-604.

11. Sanchis J, Gich I, Pedersen S. Systematic review of errors in inhaler use. Chest 2016:150:394-406.

12. Broeders ME, Molema J, Hop WC, et al. Inhalation profiles in asthmatics and COPD patients: reproducibility and effect of instruction. J Aerosol Med 2003;16:131-41.

13. Brocklebank $D$, Ram F, Wright J, et al. Comparison of the effectiveness of inhaler devices in asthma and chronic obstructive airways disease: a systematic review of the literature. Health Technol Assess 2001;5:1-149.

14. Dolovich MB, Ahrens RC, Hess DR, et al. Device selection and outcomes of aerosol therapy: Evidence-based guidelines: American college of chest physicians/american college of asthma, allergy, and immunology. Chest 2005;127:335-71.

15. Giraud V, Roche N. Misuse of corticosteroid metered-dose inhaler is associated with decreased asthma stability. Eur Respir $J$ 2002;19:246-51.

16. Melani AS, Bonavia M, Cilenti $\mathrm{V}$, et al. Inhaler mishandling remains common in real life and is associated with reduced disease control. Respir Med 2011;105:930-8.

17. Roggeri A, Micheletto C, Roggeri DP. Inhalation errors due to device switch in patients with chronic obstructive pulmonary disease and asthma: critical health and economic issues. Int $J$ Chron Obstruct Pulmon Dis 2016;11:597-602.

18. Price DB, Román-Rodríguez M, McQueen RB, et al. Inhaler Errors in the CRITIKAL Study: Type, Frequency, and Association with Asthma Outcomes. J Allergy Clin Immunol Pract 2017;5.

19. Usmani OS, Lavorini F, Marshall J, et al. Critical inhaler errors in asthma and COPD: a systematic review of impact on health outcomes. Respir Res 2018;19:10.

20. Laube BL, Janssens HM, de Jongh $\mathrm{FH}$, et al. What the pulmonary specialist should know about the new inhalation therapies. Eur Respir J 2011;:37:1308-31.

21. Tommelein E, Mehuys E, Van Hees T, et al. Effectiveness of pharmaceutical care for patients with chronic obstructive pulmonary disease (PHARMACOP): a randomized controlled trial. Br J Clin Pharmacol 2014;77:756-66.

22. Takemura M, Mitsui K, Ido M, et al. Effect of a network system for providing proper inhalation technique by community pharmacists on clinical outcomes in COPD patients. Int J Chron Obstruct Pulmon Dis 2013;8:239-44.

23. Broeders ME, Sanchis J, Levy ML, et al. The ADMIT series-issues in inhalation therapy. 2. Improving technique and clinical effectiveness. Prim Care Respir J 2009;18:76-82. 
24. Crompton GK, Barnes PJ, Broeders M, et al. The need to improve inhalation technique in Europe: a report from the Aerosol Drug Management Improvement Team. Respir Med 2006;100:1479-94.

25. Allen SC, Jain M, Ragab S, et al. Acquisition and short-term retention of inhaler techniques require intact executive function in elderly subjects. Age Ageing 2003;32:299-302.

26. Barrons R, Pegram A, Borries A. Inhaler device selection: special considerations in elderly patients with chronic obstructive pulmonary disease. Am J Health Syst Pharm 2011;68:1221-32.

27. Quinet $P$, Young CA, Héritier F. The use of dry powder inhaler devices by elderly patients suffering from chronic obstructive pulmonary disease. Ann Phys Rehabil Med 2010;53:69-76.

28. Allen SC, Warwick-Sanders M, Baxter M. A comparison of four tests of cognition as predictors of inability to learn to use a metered dose inhaler in old age. Int J Clin Pract 2009;63:1150-3.

29. Blasi F, Canonica GW, Centanni S, et al. Genuair usability test: Results of a national public survey of the elderly. COPD 2016:13:367-71.

30. Rootmensen GN, van Keimpema AR, Looysen EE, et al. The effects of additional care by a pulmonary nurse for asthma and COPD patients at a respiratory outpatient clinic: results from a double blind, randomized clinical trial. Patient Educ Couns 2008;70:179-86.

31. Bourbeau J, Julien M, Maltais F, et al. Reduction of hospital utilization in patients with chronic obstructive pulmonary disease: a disease-specific self-management intervention. Arch Intern Med 2003;163:585-91.

32. Khdour MR, Kidney JC, Smyth BM, et al. Clinical pharmacy-led disease and medicine management programme for patients with COPD. Br J Clin Pharmacol 2009;68:588-98.

33. Strayhorn-Smith V, Tolley E, Demirkan K, et al. Metered dose inhaler - spacer technique in hospitalized geriatric patients: Effect of patient education by a pharmacist. Hosp Pharm 2000;35:162-4.

34. Normansell R, Kew KM, Mathioudakis AG. Interventions to improve inhaler technique for people with asthma. Cochrane Database Syst Rev 2017;3:CD012286.

35. Thomas RM, Locke ER, Woo DM, et al. Inhaler Training Delivered by Internet-Based Home Videoconferencing Improves Technique and Quality of Life. Respir Care 2017;62:1412.

36. Press VG, Kelly CA, Kim JJ, et al. Virtual teach-to-goal adaptive learning of inhaler technique for inpatients with asthma or COPD. $J$ Allergy Clin Immunol Pract 2017;5:1032-9.

37. Wu M, Woodrick NM, Arora VM, et al. Developing a virtual teach-togoa inhaler technique learning module: A mixed methods approach. $J$ Allergy Clin Immunol Pract 2017;5:1728-36.

38. Bosnic-Anticevich SZ, Sinha H, So S, et al. Metered-dose inhaler technique: the effect of two educational interventions delivered in community pharmacy over time. J Asthma 2010;47:251-6.

39. Crane MA, Jenkins CR, Goeman DP, et al. Inhaler device technique can be improved in older adults through tailored education: findings from a randomised controlled trial. NPJ Prim Care Respir Med 2014;24:14034

40. Lavorini F, Levy ML, Corrigan C, et al. The ADMIT series - issues in inhalation therapy. 6) Training tools for inhalation devices. Prim Care Respir J 2010;19:335-41.

41. Press VG, Arora VM, Shah LM, et al. Misuse of respiratory inhalers in hospitalized patients with asthma or COPD. J Gen Intern Med 2011;26:635-42.

42. Paasche-Orlow MK, Riekert KA, Bilderback A, et al. Tailored education may reduce health literacy disparities in asthma selfmanagement. Am J Respir Crit Care Med 2005;172:980-6.
43. Self TH, Hoth LM, Bolin JM, et al. Metered-dose inhaler technique per the global initiative for asthma and expert panel report 3. Annals of Allergy, Asthma \& Immunology 2016;117:101-2.

44. Göris S, Tasci S, Elmali F. The effects of training on inhaler technique and quality of life in patients with COPD. J Aerosol Med Pulm Drug Deliv 2013;26:336-44.

45. GINA. Pocket Guide for Asthma Management and Prevention. 2015:32. Updated http://ginasthma.org.

46. GOLD. Pocket Guide to COPD Diagnosis, Management, and prevention - a guide for health care professionals. 2017. report:42 http://www.goldcopd.org.

47. Fonseca JA, Nogueira-Silva L, Morais-Almeida M, et al. Validation of a questionnaire (CARAT10) to assess rhinitis and asthma in patients with asthma. Allergy 2010;65:1042-8.

48. Roxo JP, Ponte EV, Ramos DC, et al. [Portuguese-language version of the Asthma Control Test]. J Bras Pneumol 2010;36:159-66.

49. Camelier A, Rosa FW, Salim C, et al. Using the Saint George's Respiratory Questionnaire to evaluate quality of life in patients with chronic obstructive pulmonary disease: validating a new version for use in Brazil. J Bras Pneumol 2006;32:114-22.

50. Faria da Silva LS. Validação do questionário clínico para a doença pulmonar obstructiva crónica (CCQ) para a Língua Portuguesa [Master Thesis]. Escola Superior de Enfermágem do Porto 2012.

51. Ferreira J, Silveira P, Figueiredo MM, et al. Validation of the Portuguese version of the Asthma Quality of Life Questionnaire [AQLQ-M] by Marks. Rev Port Pneumol 2005;11:351-66.

52. Ben AJ, Neumann CR, Mengue SS. The Brief Medication Questionnaire and Morisky-Green test to evaluate medication adherence. Rev Saude Publica 2012;46:279-89.

53. (ADMIT) TADMIT. Inhalation Protocols. Secondary Inhalation Protocols, 2017.

54. Silva GP, Morano MT, Viana CM, et al. Portuguese-language version of the COPD Assessment Test: validation for use in Brazil. $J$ Bras Pneumol 2013;39:402-8.

55. Kovelis D, Segretti NO, Probst VS, et al. Validation of the modified pulmonary functional status and dyspnea questionnaire and the medical research council scale for use in brazilian patients with chronic obstructive pulmonary disease. J Bras Pneumol 2008;34:1008-18.

56. Amaro F. Escala de Graffar, 1990.

57. Apóstolo JLA, LMdJ L, IACd R, et al. Contribuição para a adaptação da Geriatric Depression Scale -15 para a língua portuguesa. Revista de Enfermagem Referência 2014:65-73. serIV.

58. Nunes DP, Duarte YA, Santos JL, et al. Screening for frailty in older adults using a self-reported instrument. Rev Saude Publica 2015:49:2.

59. Freitas Sandra SMR, Cristina M, Manuela V, et al. Estudos de adaptação do Montreal Cognitive Assessment (MoCA) para a população portuguesa. Aval. psicol. [online] 2010;9:345-57.

60. Suissa S. Statistical treatment of exacerbations in therapeutic trials of chronic obstructive pulmonary disease. Am J Respir Crit Care Med 2006;173:842-6.

61. Bland JM, Altman DG. Multiple significance tests: the Bonferroni method. BMJ 1995;310:170.

62. Moran C, Doyle F, Sulaiman I, et al. The INCA(TM) (Inhaler Compliance Assessment(TM)): A comparison with established measures of adherence. Psychol Health 2017;32:1266-87. 